\title{
Three-Body Halo Fragmentation: Polarization effects
}

\author{
E. Garrido, D.V. Fedorov and A.S. Jensen \\ Institute of Physics and Astronomy, \\ Aarhus University, DK-8000 Aarhus C, Denmark
}

(May 17, 2018)

\begin{abstract}
Momentum distributions of particles from nuclear break-up of fast three-body halos are calculated for polarized beam and outgoing fragments. These momentum distributions are combined in observable quantities which emphasize the two-body correlations in the initial three-body structure as well as in the final two-body system. Applying the formalism to ${ }^{11} \mathrm{Li}$ we find a sensitive dependence on the $p$-wave content and on the position of the $p$-resonances in ${ }^{10} \mathrm{Li}$. Polarization experiments are then a new tool to study correlations in nuclear halos.
\end{abstract}

PACS. 25.60.+v - Reactions induced by unstable nuclei.

PACS. 24.70.+s - Polarization phenomena in reactions.

PACS. 21.45.+v - Few-body systems.

Introduction. The unusual halo nuclei appear under conditions realized at the neutron dripline [1]. The structure of these nuclei is dominated by a configuration where an ordinary nucleus (core) is surrounded by weakly bound neutrons. For Borromean systems [2.3], three particles without bound two-body subsystems, specific correlations must be present to provide the total binding. The nature of these correlations is rather difficult to investigate and the data have almost always been consistent with uncorrelated motion of particles around the core. The mixture of $s$ - and $p$-states in the neutron-core subsystem is an obvious manifestation of the correlations. However its existence has been rather difficult to confirm experimentally.

The major source of detailed information is the measurements of particle momentum distributions after fragmentation reactions of these halo nuclei 细 6 . In the typical experimental setup two of the three constituent particles are detected after the high energy halo fragmentation in anticoincidence with the third particle, which has been instantly captured by the target. The sudden approximation or the Serber mechanism, equivalent to instantaneous cease of the interactions between the constituent particles, has been proved to be adequate in data interpretation [3, 8]. Furthermore, it has been pointed out recently that inclusion of the interaction between the two remaining particles greatly improves the model [5, 9.10].

A polarized beam interacting with a target is a tool sensitive to components of the wave function with non-zero relative angular momentum. In particular for ${ }^{11} \mathrm{Li}$, the resulting particle momentum distribution should be sensitive to the amount of $p$-state admixture in the neutron-core relative state. More details and strong signals are expected when the polarization of the fragments is simultaneously measured.

The purpose of this letter is to investigate the effects of polarization on halo nuclear reactions in view of extracting information about the correlations in these nuclei. We shall first briefly sketch the new method for computation of fragment momentum distributions arising from polarized beam experiments. We shall investigate the effects of polarized beams and unpolarized fragments, as well as polarized beams and polarized fragments.

Method. In the sudden approximation the transition matrix is proportional to the overlap between the initial and the final wave functions. This approximation is suitable for neutron halo fragmentation, as first suggested for the deuteron stripping by Serber [11] and later used in many connections [12]. The projectile wave function is denoted by $\Psi^{J M}(\mathbf{x}, \mathbf{y})$, where $J$ and $M$ are the total spin and its projection. The coordinates $\mathbf{x}$ and $\mathbf{y}$ are the usual Jacobi coordinates [2], where $\mathbf{x}$ is drawn between the two particles surviving after the fragmentation. The final state wave function is given by $e^{i \mathbf{k}_{y} \cdot \mathbf{y}} \chi_{s_{y}}^{\sigma_{y}} \sum_{s_{x} \sigma_{x}}\left\langle s_{1}, s_{2} ; \sigma_{1}, \sigma_{2} \mid s_{x} \sigma_{x}\right\rangle w^{s_{x} \sigma_{x}}\left(\mathbf{k}_{x}, \mathbf{x}\right)$, where $w^{s_{x} \sigma_{x}}\left(\mathbf{k}_{x}, \mathbf{x}\right)$ describes the final two-body system with spin $s_{x}$ and projection $\sigma_{x}$. The quantum numbers $s_{1}, s_{2}, y, \sigma_{1}, \sigma_{2}$, and $\sigma_{y}$ are the spins and projections of the three particles, and the plane wave describes the motion of the center of mass of the two-body system. The plane waves [3] correctly describe the shapes of the high energy momentum distributions in the region of the first maximum. By squaring this overlap, and subsequently summing up over the initial and final magnetic substates we get the following expression for the cross section or momentum distribution

$$
\begin{aligned}
\frac{d^{6} \sigma}{d \mathbf{k}_{x} d \mathbf{k}_{y}} \propto & \sum_{M} W_{\mathrm{init}}(M) \sum_{\sigma_{1}} W_{\mathrm{fin}}\left(\sigma_{1}\right) \sum_{\sigma_{2}} W_{\mathrm{fin}}\left(\sigma_{2}\right) \sum_{s_{x}, s_{x}^{\prime}, \sigma_{x}}\left\langle s_{1}, s_{2} ; \sigma_{1}, \sigma_{2} \mid s_{x} \sigma_{x}\right\rangle\left\langle s_{1}, s_{2} ; \sigma_{1}, \sigma_{2} \mid s_{x}^{\prime} \sigma_{x}\right\rangle \\
& \sum_{\sigma_{y}}\left\langle e^{i \mathbf{k}_{y} \cdot \mathbf{y}} \chi_{s_{y}}^{\sigma_{y}} w^{s_{x} \sigma_{x}}\left(\mathbf{k}_{x}, \mathbf{x}\right) \mid \Psi^{J M}(\mathbf{x}, \mathbf{y})\right\rangle^{*}\left\langle e^{i \mathbf{k}_{y} \cdot \mathbf{y}} \chi_{s_{y}}^{\sigma_{y}} w^{s_{x}^{\prime} \sigma_{x}}\left(\mathbf{k}_{x}, \mathbf{x}\right) \mid \Psi^{J M}(\mathbf{x}, \mathbf{y})\right\rangle
\end{aligned}
$$


where $W_{\text {init }}(M), W_{\text {fin }}\left(\sigma_{1}\right)$, and $W_{\text {fin }}\left(\sigma_{2}\right)$ give the probability of occupation of each magnetic substate in the initial and final states. When the beam is not polarized and there is no detection of final spin states these probabilities take the values $1 /(2 J+1), 1 /\left(2 s_{1}+1\right)$, and $1 /\left(2 s_{2}+1\right)$, and we obtain the unpolarized expression.

From $\mathbf{x}$ and $\mathbf{y}$ we define the hyperspherical coordinates $\rho=\sqrt{x^{2}+y^{2}}, \alpha=\arctan (x / y)$, and $\Omega_{x}$ and $\Omega_{y}$ giving the directions of $\mathbf{x}$ and $\mathbf{y}$. Analogously, in momentum space we define $\kappa=\sqrt{k_{x}^{2}+k_{y}^{2}}, \alpha_{\kappa}=\arctan \left(k_{x} / k_{y}\right), \Omega_{k_{x}}$, and $\Omega_{k_{y}}$.

The three-body wave function is obtained by solving the Faddeev equations in coordinate space [13]. This function is then expanded in terms of the complete set of hyperspherical harmonics $\mathbf{Y}_{\ell_{x}, \ell_{y}}^{K, L}\left(\alpha, \Omega_{x}, \Omega_{y}\right)$, where $\ell_{x}$ and $\ell_{y}$ are the orbital angular momenta associated to the Jacobi coordinates $\mathbf{x}$ and $\mathbf{y}$, and $L$ is the coupling of both momenta. The quantum number $K$ is usually called the hypermoment. The function $\Psi^{J M}(\mathbf{x}, \mathbf{y})$ can then be expanded as

$$
\Psi^{J M}(\mathbf{x}, \mathbf{y})=\sum_{K \ell_{x} \ell_{y} s_{x} L S} F_{\ell_{x} \ell_{y} s_{x}}^{K L S}(\rho)\left[\mathbf{Y}_{\ell_{x}, \ell_{y}}^{K, L}\left(\alpha, \Omega_{x}, \Omega_{y}\right) \otimes \chi_{s_{x}, s_{y}}^{S}\right]^{J M}
$$

where $\chi_{s_{x}, s_{y}}^{S}$ is the spin function obtained by coupling the two-body spin $s_{x}$ and the spin $s_{y}$ of the third particle to the total spin $S$.

We expand $w^{s_{x} \sigma_{x}}\left(\mathbf{k}_{x}, \mathbf{x}\right)$ in partial waves

$$
w^{s_{x} \sigma_{x}}\left(\mathbf{k}_{x}, \mathbf{x}\right)=\sqrt{\frac{2}{\pi}} \frac{1}{k_{x} x} \sum_{j_{x}, \ell_{x}, m_{x}} u_{\ell_{x} s_{x}}^{j_{x}}\left(k_{x}, x\right) \mathcal{Y}_{j_{x} \ell_{x} s_{x}}^{m_{x}^{*}}\left(\Omega_{x}\right) \sum_{m_{\ell_{x}}}\left\langle\ell_{x} s_{x} ; m_{\ell_{x}} \sigma_{x} \mid j_{x} m_{x}\right\rangle i_{x}^{\ell} Y_{\ell_{x} m_{\ell_{x}}}\left(\Omega_{k_{x}}\right),
$$

where the radial functions $u_{\ell_{x} s_{x}}^{j_{x}}\left(k_{x}, x\right)$ are obtained by solving the Schrödinger equation with the corresponding twobody potential. The quantum numbers contained in eq.(3) correspond to the ones in the two-body subsystem in eq.(2). Following this procedure we incorporate Final State Interactions (FSI) into the calculation. When FSI is not considered the function $w^{s_{x} \sigma_{x}}\left(\mathbf{k}_{x}, \mathbf{x}\right)$ reduces to a plane wave, and the overlaps in eq.(1) become the Fourier transform of the three-body wave function.

First we consider the case of $100 \%$ polarized beam $\left(W_{\text {init }}\left(M^{\prime}\right)=\delta_{M^{\prime} M}\right)$. After substituting eqs.(2) and (3) into eq.(11), and integrating analytically over $\Omega_{k_{y}}$ and $\varphi_{k_{x}}\left(\Omega_{k_{x}} \equiv\left(\theta_{k_{x}}, \varphi_{k_{x}}\right)\right)$, we get the expression

$$
\begin{aligned}
& \frac{d^{3} \sigma}{d k_{y} d k_{x}^{\perp} d k_{x}^{\|}} \propto k_{y}^{2} k_{x}^{\perp} \frac{2}{\pi^{2}} \sum_{I}(-1)^{J-M}\left(\begin{array}{ccc}
J & J & I \\
M & -M & 0
\end{array}\right) \hat{I}^{2} P_{I}\left(\cos \theta_{k_{x}}\right) \sum_{j_{x} \ell_{x} s_{x}} \sum_{j_{x}^{\prime} j_{y} \ell_{y}} \sum_{L S L^{\prime} S^{\prime}} \\
&(-1)^{2 s_{y}+J-s_{x}+j_{x}-j_{x}^{\prime}-j_{y}} \mathcal{I}_{\ell_{x} \ell_{x} j_{x}}^{\ell_{L} S}\left(\kappa, \alpha_{\kappa}\right) \mathcal{I}_{\ell_{x} s_{x} \bar{L}_{x}^{\prime} S_{x}^{\prime}}\left(\kappa, \alpha_{\kappa}\right) \hat{j}_{x}^{2} \hat{j}^{\prime}{ }_{x}^{2} \hat{j}_{y}^{2} \hat{\ell}_{x}^{2} \hat{J}^{2} \hat{L} \hat{S} \hat{L}^{\prime} \hat{S}^{\prime} \\
&\left(\begin{array}{ccc}
\ell_{x} & \ell_{x} & I \\
0 & 0 & 0
\end{array}\right)\left\{\begin{array}{ccc}
j_{x} & j_{x}^{\prime} & I \\
J & J & j_{y}
\end{array}\right\}\left\{\begin{array}{lll}
j_{x} & j_{x}^{\prime} & I \\
\ell_{x} & \ell_{x} & s_{x}
\end{array}\right\}\left\{\begin{array}{ccc}
J & j_{x} & j_{y} \\
L & \ell_{x} & \ell_{y} \\
S & s_{x} & s_{y}
\end{array}\right\}\left\{\begin{array}{ccc}
J & j_{x}^{\prime} & j_{y} \\
L^{\prime} & \ell_{x} & \ell_{y} \\
S^{\prime} & s_{x} & s_{y}
\end{array}\right\}
\end{aligned}
$$

where $k_{y}=\left|\mathbf{k}_{y}\right|, k_{x}^{\perp}=\left|\mathbf{k}_{x}\right| \sin \theta_{k_{x}}, k_{x}^{\|}=\left|\mathbf{k}_{x}\right| \cos \theta_{k_{x}}$, and $\hat{a}=\sqrt{2 a+1}$. The functions $\mathcal{I}_{\ell_{x} s_{x} L S}\left(\kappa, \alpha_{\kappa}\right)$ are only available as numerical functions. By integration over $k_{y}$ and either $k_{x}^{\perp}$ or $k_{x}^{\|}$, we obtain respectively $\mathcal{Q}_{1}^{M}\left(k_{x}^{\|}\right)$or $\mathcal{Q}_{2}^{M}\left(k_{x}^{\perp}\right)$, the one-dimensional or two-dimensional momentum distribution (note that in our description there is no preferred axis, and therefore the one-dimensional longitudinal and transverse momentum distributions are identical).

From the definitions of the coordinates it is clear that $\mathcal{Q}_{1,2}^{M}$ expresses the relative momentum distribution of the two particles in the final state. To compare with the experimental data one has to refer the momentum distributions to the center of mass of the three-body system. The appropriate expressions, used in the numerical calculations, are rather complicated but the qualitative features remain unchanged. Details about these expressions will be given in a forthcoming paper.

The summation over $M$ in eq.(何) leads to the unpolarized momentum distribution, that amounts to keeping only the $I=0$ term. The terms with $I \neq 0$ appear only when the beam is polarized, and only $\ell_{x} \neq 0$ waves contribute.

We now define the asymmetry

$$
A_{i}^{M M^{\prime}}=\frac{\mathcal{Q}_{i}^{M}-\mathcal{Q}_{i}^{M^{\prime}}}{\sum_{M} \mathcal{Q}_{i}^{M}}, \quad i=1,2
$$

that vanishes when the beam is not polarized. The deviations from zero are then produced by the $I \neq 0$ terms in $\mathcal{Q}_{i}^{M}$, or, in other words, by the $\ell_{x} \neq 0$ waves contained in the neutron-core subsystem. 
Finally, together with a polarized beam, we consider the possibility of measuring the polarization of one of the fragments in the final state. Then the momentum distribution is similar to eq.(4), but containing additional geometrical factors involving the $z$-projection $\sigma$ of the spin of the polarized particle in the final state (the expressions are rather extended, and the details will be published in a coming paper). From the one and two-dimensional momentum distributions $\mathcal{Q}_{1}^{M \sigma}\left(k_{x}^{\|}\right)$and $\mathcal{Q}_{2}^{M \sigma}\left(k_{x}^{\perp}\right)$ we then introduce the asymmetry

$$
A_{i}^{M M^{\prime} ; \sigma \sigma^{\prime}}=\frac{\mathcal{Q}_{i}^{M \sigma}-\mathcal{Q}_{i}^{M^{\prime} \sigma^{\prime}}}{\sum_{M \sigma} \mathcal{Q}_{i}^{M \sigma}}, \quad i=1,2
$$

where again the deviations from zero are produced by the polarizations. However, in this case also $s$-waves contribute.

Results. We apply the method to the three-body halo ${ }^{11} \mathrm{Li}\left({ }^{9} \mathrm{Li}+\mathrm{n}+\mathrm{n}\right)$ fragmentation, where one of the neutrons is suddenly captured by the target. The two-body potentials used in the calculations are given in ref. [13], where the nucleon-nucleon potential is fitted to low energy $s$ - and $p$-wave nucleon-nucleon scattering data, and the neutroncore potential is adjusted to reproduce the experimental binding energy and mean square radius of the three-body system. In all the calculations the lowest virtual $s$ state in ${ }^{10} \mathrm{Li}$ is at $50 \mathrm{keV}$, and the lowest $p$-resonance is at 0.5 $\mathrm{MeV}$, according to the experimental data [5]. The rest of the resonances occurs at different energies, modifying the p-content in the two-body subsystem and keeping the right three-body binding energy.

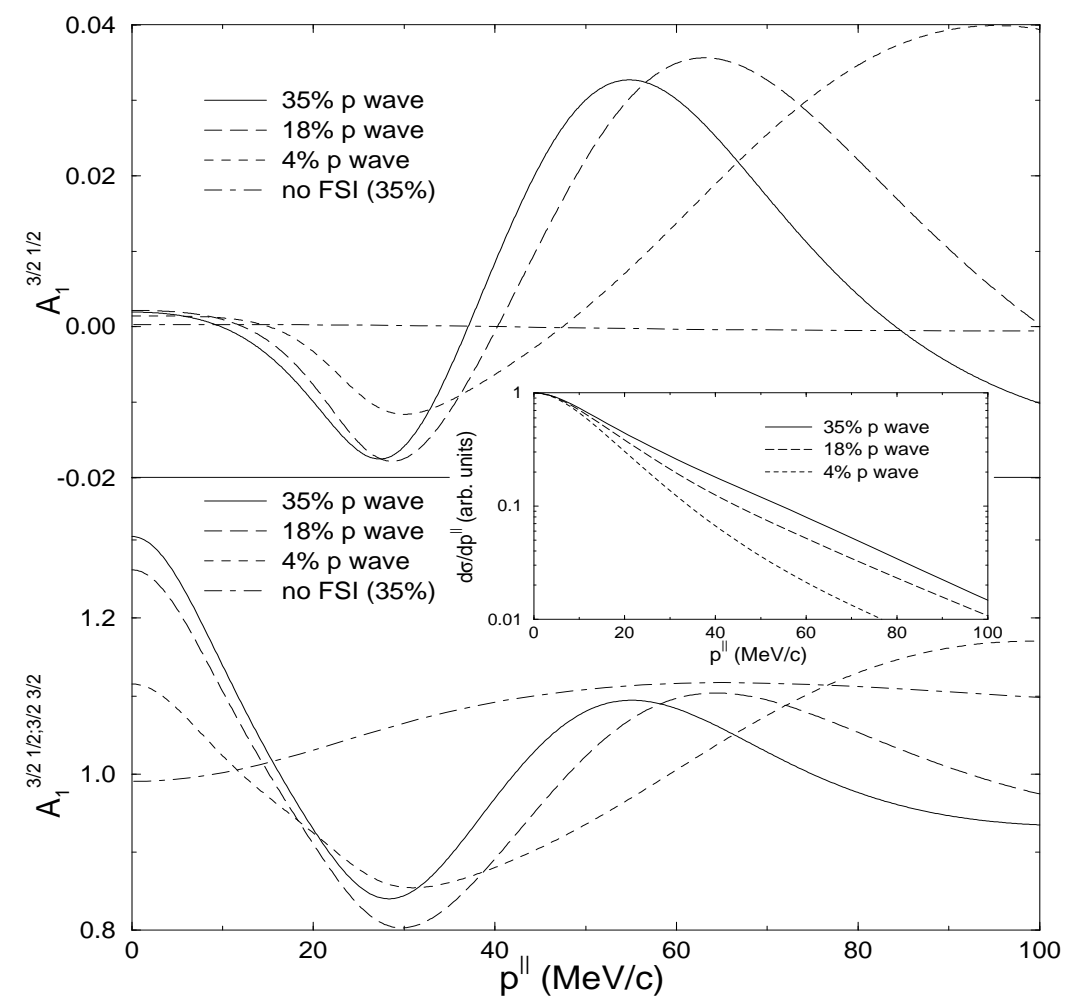

FIG. 1. Upper part: Asymmetry $A_{1}^{\frac{3}{2}} \frac{1}{2}$ for neutron momentum distributions from ${ }^{11} \mathrm{Li}$ fragmentation reaction with polarized beam. Three different contents of $p$-wave in the neutron-core system are considered: $35 \%$ (solid), 18\% (long dashed), and $4 \%$ (dashed). The curve for $35 \%$ of $p$-wave without final state interactions (dot-dashed) is also shown. Lower part: The same as in the upper part for the asymmetry $A_{1}^{\frac{3}{2} \frac{1}{2} ; \frac{3}{2} \frac{3}{2}}$. Inner part: Unpolarized one-dimensional neutron momentum distribution from neutron removal process in ${ }^{11} \mathrm{Li}$ fragmentation. The neutron momentum $p$ is relative to the center of mass of the three-body system.

Let us first focus on the asymmetry $A_{1}^{\frac{3}{2}} \frac{1}{2}$ for momentum distributions of the surviving neutron. This asymmetry is shown in the upper part of fig. 1 , where three curves corresponding to different $p$-wave content in the neutron- ${ }^{9} \mathrm{Li}$ channel $(4,18$, and $35 \%)$ are shown.

From this part of the figure we can conclude the following:

(i) The deviations from zero of the asymmetry are produced by the $p$-waves in the neutron- ${ }^{9} \mathrm{Li}$ subsystem. A significant dependence on the $p$-wave content of the two-body wave function is observed. Since the asymmetry is zero when no $p$-waves are present, one could expect smaller oscillations for small $p$-wave content. However, $p$-waves 
are also contained in the unpolarized momentum distribution, in such a way that the less the $p$-wave content, the narrower the momentum distribution (see the inner part of fig.1). Therefore the denominator of the asymmetry tends to increase the amplitude of the oscillations for low $p$-wave content in the two-body wave function. It is then possible, as in fig.1, to have oscillations with similar amplitude for different values of the $p$-wave content.

(ii) If the two-body system has a resonance at a given energy $E_{\text {res }}$, then the enhanced overlaps in eq.(1) produce a bump in the momentum distribution at $k=\sqrt{2 \mu E_{\text {res }}}$ ( $\mu$ is the reduced mass of the two particles). For the same reason the difference between $\mathcal{Q}_{i}^{M}$ and the unpolarized momentum distribution is expected to be larger in the regions close to the resonances in the two-body system. Therefore the asymmetry should present larger oscillations for the momentum values corresponding to $p$-resonances in ${ }^{10} \mathrm{Li}$. In particular, the $p$-resonance at $0.5 \mathrm{MeV}$ corresponds to a momentum of $30 \mathrm{MeV} / \mathrm{c}$, that matches with the position of the first peak in the asymmetries. The second $p$-resonance changes its position from case to case, being placed at $1.5 \mathrm{MeV}(k \sim 50 \mathrm{MeV} / \mathrm{c})$ for the solid curve, $2 \mathrm{MeV}(k \sim 60$ $\mathrm{MeV} / \mathrm{c})$ for the long-dashed curve, and $6 \mathrm{MeV}(k \sim 100 \mathrm{MeV} / \mathrm{c})$ for the dashed line. From the figure it is then clear that the asymmetry gives an indication about the level structure of ${ }^{10} \mathrm{Li}$.

(iii) If we consider the three particles in the initial state coupled to total orbital angular momentum $L=0$, the polarization of the beam affects only the spin part of the wave function. If then none of the final spin projections are measured, neglecting the interaction between ${ }^{9} \mathrm{Li}$ and the remaining neutron (no FSI) leads to momentum distributions that are equal to the unpolarized ones, and the asymmetry therefore vanishes (this can be seen analytically by inserting eqs.(2) and (3) into eq.(11) for $L=0$ ). In our case, the ${ }^{11} \mathrm{Li}$ wave function contains more than $95 \%$ of $L=0$ configuration, and therefore the asymmetry neglecting FSI is almost zero (see the dot-dashed line in the figure). We can therefore conclude that FSI is necessary for the correct description of the asymmetry.

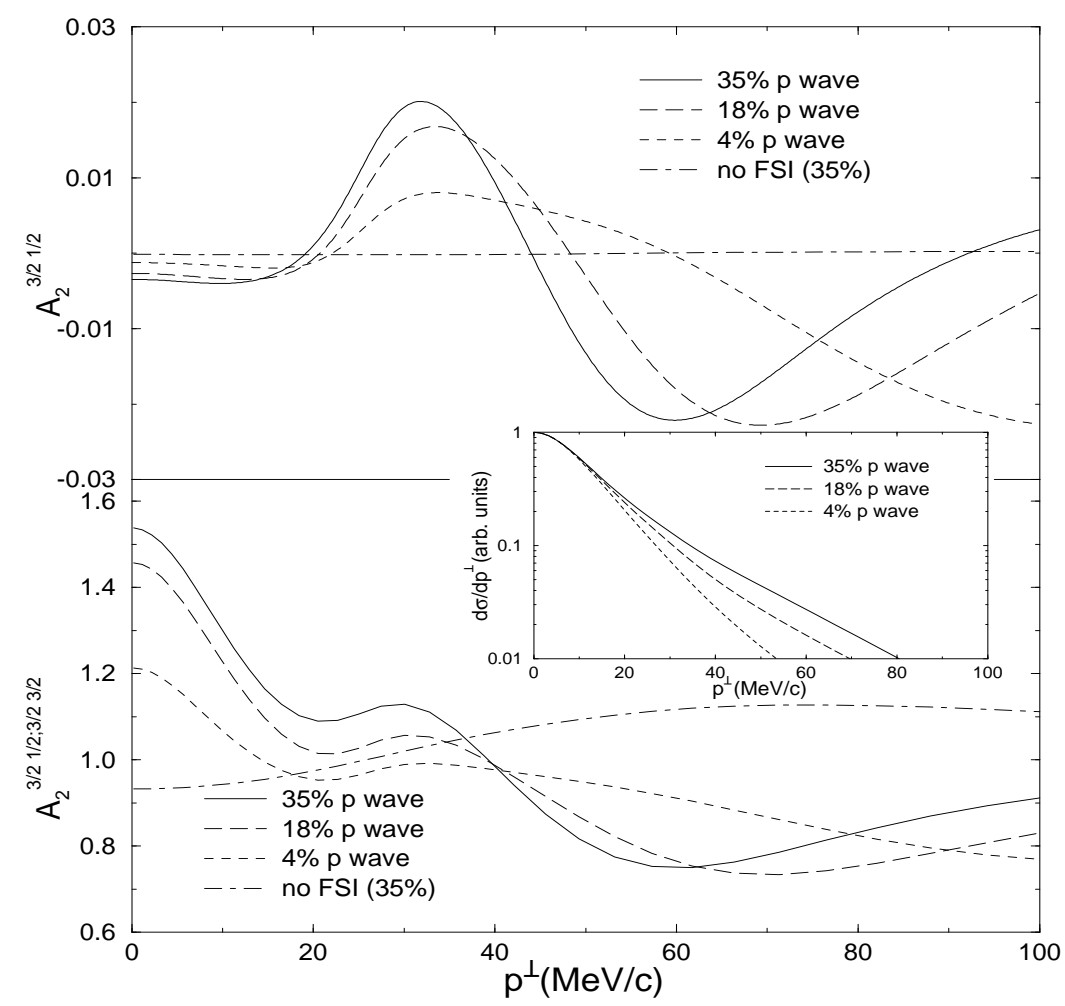

FIG. 2. The same as in fig.1 for two-dimensional neutron momentum distributions.

We turn now to the case where the polarization of the surviving core also is measured. We assume that the core is longitudinally polarized and consider the asymmetry $A_{1}^{\frac{3}{2}} \frac{1}{2} ; \frac{3}{2} \frac{3}{2}$. In the lower part of fig. 1 we plot this asymmetry for the same three cases we considered above. The three points we already emphasized are valid again. First a clear dependence on the $p$-wave content is observed even though the $s$-waves also appear in the numerator of the asymmetry. The effect of $s$-waves is however confined to the low momentum region, and for momenta larger than $20 \mathrm{MeV} / \mathrm{c}$ only $p$-waves contribute. Therefore the minima and maxima of the asymmetry again match with the $p$-resonances in ${ }^{10} \mathrm{Li}$. Finally the dot-dashed line shows that FSI is also essential in this case. However as the polarization of the core is now measured, even $L=0$ terms in the three-body wave function contribute to the asymmetry. This asymmetry is 
then an observable with similar characteristics as $A_{1}^{\frac{3}{2}} \frac{1}{2}$, but with the advantage that the oscillations are larger, and therefore easier to observe experimentally.

In fig. 2 we show the same calculations as in fig.1, but for two-dimensional neutron momentum distributions. The curves show features similar to the curves in fig.1. Our conclusions about the behaviour of the asymmetries are therefore rather general and not restricted to only one kind of momentum distributions.

Summary and conclusions. The aim of this letter is to investigate polarizations in three-body halo fragmentation reactions at high energies and light targets. We use the sudden approximation where the initial state is a genuine three-body wave function and the final state is a distorted wave in the same potential between the two surviving particles as used in the three-body calculation. We have defined several asymmetries from which the polarization effects can be analyzed.

We applied the method to ${ }^{11} \mathrm{Li}$ fragmentation, and computed one and two-dimensional neutron momentum distributions. We have found that the polarization observables are more sensitive to the $p$-wave content in the neutron-core subsystem than unpolarized cross sections. Furthermore they provide a unique information of the level structure of the two-body subsystem. We also observed that FSI is the main cause for the behaviour of the asymmetries, and FSI must necessarily be included in the calculation. These features have been found for fragmentation reactions with polarized beam, and also when simultaneously the polarization of the core is measured. The advantage of the second case is that the amplitude of the oscillations observed in the asymmetries is clearly larger. Finally we have shown that these conclusions are valid for both one and two-dimensional momentum distributions.

Acknowledgments One of us (E.G.) acknowledges support from the European Union through the Human Capital and Mobility program contract nr. ERBCHBGCT930320.

[1] P.G. Hansen, A.S. Jensen, and B. Jonson, Ann. Rev. Nucl. Part. Sci., 45 (1995) 591.

[2] D.V. Fedorov, A.S. Jensen, and K. Riisager, Phys. Rev. C, 49 (1994) 201.

[3] M.V. Zhukov, B.V. Danilin, D.V. Fedorov, J.M. Bang, I.J. Thompson, and J.S. Vaagen, Phys. Rep., 231 (1993) 151.

[4] R. Anne et al., Phys. Lett. B, 250 (1990) 19.

[5] M. Zinser et al., Phys. Rev. Lett., 75 (1995) 1719.

[6] T. Nilsson et al., Europhys. Lett., 30 (1995) 19.

[7] F. Humbert et al., Phys. Lett. B, 347 (1995) 198.

[8] I.J. Thompson and M.V. Zhukov, Phys. Rev. C, 49 (1994) 1904.

[9] A.A. Korsheninnikov and T. Kobayashi, Nucl. Phys. A, 567 (1994) 97.

[10] E. Garrido, D.V. Fedorov, and A.S. Jensen, in press in Phys. Rev. C, 53 (1996), http://xxx.lanl.gov/abs/nuclth/9603028

[11] R. SERBER, Phys. Rev., 72 (1947) 1008.

[12] G.R. Satchler, Direct Nuclear Reactions, (Oxford University Press, Oxford, 1983).

[13] D.V. Fedorov, E. Garrido, and A.S. Jensen, Phys. Rev. C, 51 (1995) 3052. 